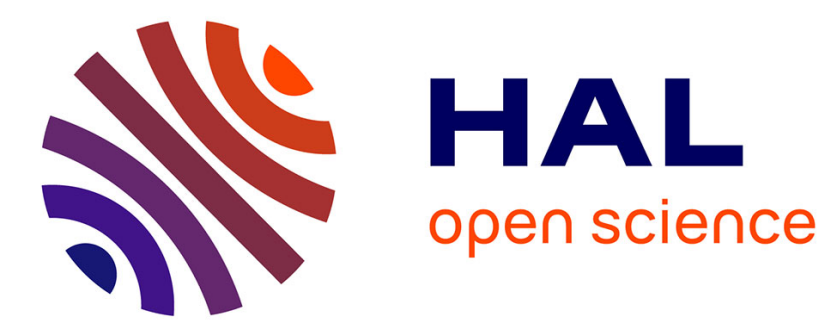

\title{
MULTISPECTRAL VENOUS IMAGES ANALYSIS FOR OPTIMUM ILLUMINATION SELECTION
}

\author{
Aamir Shahzad, Nicolas Walter, Malik Aamir Saeed, Mohammed Saad
}

Naufal, Fabrice Meriaudeau

\section{- To cite this version:}

Aamir Shahzad, Nicolas Walter, Malik Aamir Saeed, Mohammed Saad Naufal, Fabrice Meriaudeau. MULTISPECTRAL VENOUS IMAGES ANALYSIS FOR OPTIMUM ILLUMINATION SELECTION. IEEE ICIP 2013, Sep 2013, France. pp.1. hal-00860535

\section{HAL Id: hal-00860535 \\ https://hal.science/hal-00860535}

Submitted on 10 Sep 2013

HAL is a multi-disciplinary open access archive for the deposit and dissemination of scientific research documents, whether they are published or not. The documents may come from teaching and research institutions in France or abroad, or from public or private research centers.
L'archive ouverte pluridisciplinaire HAL, est destinée au dépôt et à la diffusion de documents scientifiques de niveau recherche, publiés ou non, émanant des établissements d'enseignement et de recherche français ou étrangers, des laboratoires publics ou privés. 


\title{
MULTISPECTRAL VENOUS IMAGES ANALYSIS FOR OPTIMUM ILLUMINATION SELECTION
}

\author{
A. Shahzad*, N. Walter*, Aamir Saeed Malik*, N. M. Saad*, and F. Meriaudeau ${ }^{+}$ \\ *Centre for Intelligent Signal and Imaging Research \\ Universiti Teknologi PETRONAS, Malaysia \\ +Le2i, University of Burgundy, Le Creusot, France \\ taurian_bce@yahoo.com
}

\begin{abstract}
Intravenous (IV) catheterization is the most important phase in medical practices of daily life. It is hard to localize veins in patients who have deep veins, minor age or dark skin; hence multiple attempts become indispensable for proper catheterization in such cases. Near Infrared (NIR) Imaging allow to visualize the veins underneath the skin of persons having non-visibility of veins problem. This paper reports the pre-selection of illuminants that ensure best veins/tissues contrast for patients having different skin tone. The sample subjects have been divided in four different classes based on the Luminance value of their skin tone in order to extract the best illuminant wavelengths range for each class. A multispectral approach has been used which provides the flexibility of wavelength range from visible to NIR (380 to $1040 \mathrm{~nm})$. The veins/tissue reflectance contrast obtained helps in determining the best wavelengths range where the contrast is maximum for each of the four classes. Using these results, we are planning to build a prototype system which can automatically select the illuminants based on different physiological characteristics of a subject.
\end{abstract}

Index Terms-IV Catheterization, NIR Imaging, Illuminants Subcutaneous, Absorption coefficient

\section{INTRODUCTION}

Medical treatments of today's life mostly depend on the invasive medications or blood sampling through venipuncture and IV catheterization process. These tasks are performed manually by the skilled medical professionals who either locate the veins by sight or some times on merely feelings by pressing the arm/hand of a patient with fingers. Due to many physiological differences including skin tone, veins depth, scars, presence of hair etc., the medical staff faces difficulty and stumbles in veins localization process which either increases the number of attempts and/or results in bruising or veins damage. Studies reported that 2.18 venipuncture attempts are required on average per patient who needs IV medication [1]. The patients especially infants suffer with lot of pain due to the multiple attempts for catheter insertion. A very serious consequence of unsuccessful attempt for venipuncture is the medication delivered to the tissues surrounding the IV catheter. This may result in infiltration and extravasations which may lead to surgical intervention [2].

There are few devices and techniques like Computed Tomography (CT), Positron Emission Tomography (PET), Magnetic Resonance Imaging (MRI) and Ultrasound that can be used for veins localization. Nevertheless these methods are not cost effective, bulky and are suitable only for deep veins localization. For the normal IV catheterization and blood sampling process, we are required to localize the subcutaneous veins present in hypodermis, the third layer of skin. Transillumination is a technique used to visualize the subcutaneous veins. In this technique a single wavelength from visible range of the electromagnetic spectrum is used to illuminate the targeted area of the skin [3]. Unfortunately the single visible wavelength used may not be suitable for the veins localization process due to limitation in depth penetration to the skin tissues $[4,5]$. Photoacoustic technique uses light and ultrasound waves to obtain a real time high resolution image of the blood vessels. The images obtained provide information on depth and diameter but no information is inferred about the position or shape of longitudinal or orientation of veins. Moreover this technique is highly sensitive to noise and cannot be used for venous imaging due to low signal to noise ratio (SNR) [6-8].

Near Infrared (NIR) imaging technique has vast applications in biomedical field and has several advantages over the other radiological methods used for medical spectroscopy. As the radiations are non-ionizing the technique can be applied several times on patients without any harmful effects. The noninvasive capability of the technique leads to its wide acceptance in the field of biomedical imaging. Due to low absorption coefficients of main absorbers of the skin, like hemoglobin, oxygenated hemoglobin and water in the range of 740 to $940 \mathrm{~nm}$, light rays can penetrate deeper in the skin tissues. Fig. 1 illustrates the low absorption window in the NIR range, where the absorption coefficients of these elements are in the lowest range. 


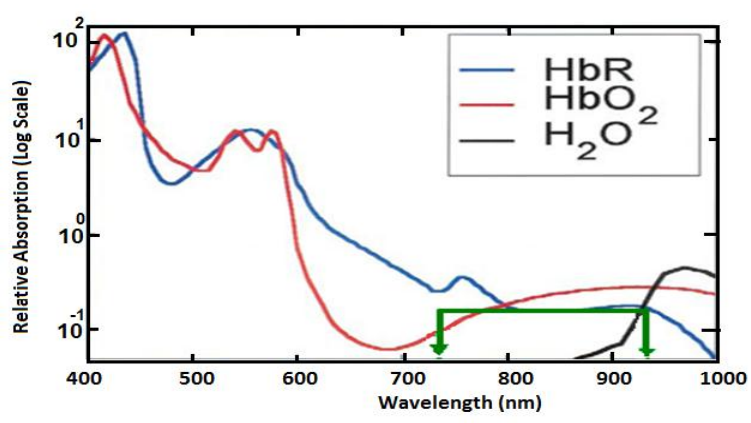

Figure 1. Absorption factors of primary absorbers: Hemoglobin, Oxygenated Hemoglobin and Water [9]. The green arrows highlight the NIR low absorption window.

So far, few devices have been reported that use NIR imaging techniques for subcutaneous veins localization. The most well-known system is the VeinViewer by Luminetx Corp [10, 11]. This device uses the wavelength of $740 \mathrm{~nm}$ and a digital NIR camera to capture the image. Image processing algorithm is used to approximate the position of veins and a binary image is projected on to the surface of the skin using green light.

This device works for average patients, but suffers to improve the quality in medical care for patients having different physiological characteristics like dark skin tone, hair, scars on the skin etc. This is due to the use of single wavelength illumination of the targeted area of skin. Determining the width of veins is the most important step for choosing vein suitable for catheterization process. The most suitable vein is the one which is wide enough for catheterization and have maximum longitudinal area. Using VeinViewer it is impossible to define the accurate measurement of the width of veins.

According to the different skin tones of patients, the use of multiple illuminants with wavelengths in NIR range will allow us to determine the wavelength combination that will maximize the contrast between skin tissues and veins [12]. The resulting system will be able to sharply visualize the width of veins. The depth of veins will be approximated by simulations using Monte Carlo method presented by Nishidate et al. in [13].

This paper presents a multispectral analysis of skin tissues and veins of different subjects to select the best combination of wavelengths (illuminants) with respect to skin tone to achieve the best veins/tissue contrast. The remainder of this paper is organized as follows: Section II will describe the data acquisition material and method. Section III will detail the theory of reflectance extraction from the multispectral images. Section IV will present the results and analysis. Finally, conclusion summarizes the research work and present future work.

\section{DATA AQUISITION}

In order to obtain a complete description of reflectance for different skin tones in visible and NIR range, a multispectral camera (Specim $\left.{ }^{\circledR}\right)$ has been used. Fig. 2 shows the setup to acquire the image of lower arm area of the subjects. The camera has the ability to acquire images in visible as well as NIR range, i.e. 400 to $1000 \mathrm{~nm}$, with a spectral resolution of $2 \mathrm{~nm}$. Images were taken under the normal room lightings. Furthermore, the projector lamps which illumination covers a spectrum from 350 to $2500 \mathrm{~nm}$ range were used as illumination devices.

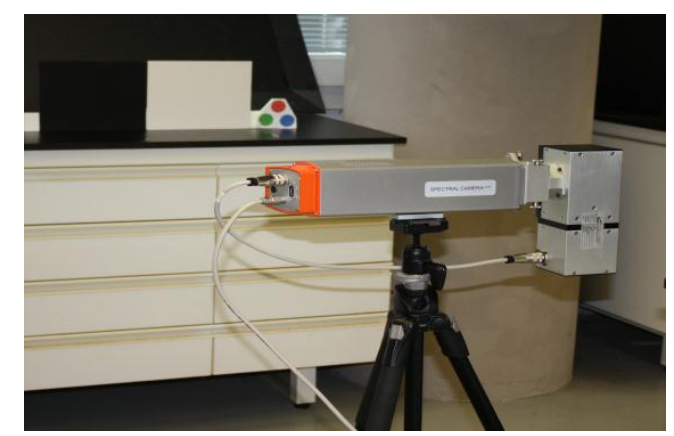

Figure 2. Image acquisition setup with white and clack reference plates.

The precise characterization of projector illumination is presented in the next section.

Skin tone of each subject is first measured using a chroma meter (Konika Minolta Inc), which provides the $L^{*} a * b$ measures of the subject's skin. Thus, the different skin tones of the subjects have been classified in function of Luminance ( $\mathrm{L}$ value) in four different classes; i.e. fair, light brown, dark brown and dark. Fig. 3 exhibits the different skin tones of subjects, highlighting the fact that veins are difficult to localize in the visible spectrum.

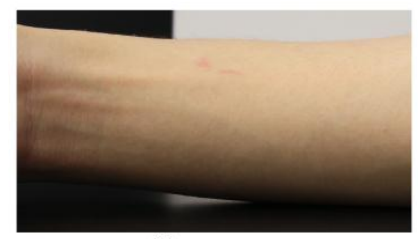

(a)

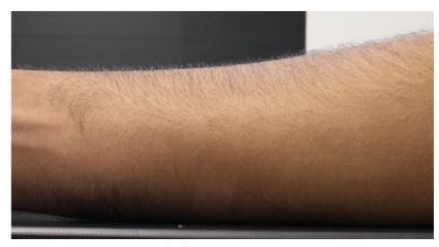

(c)

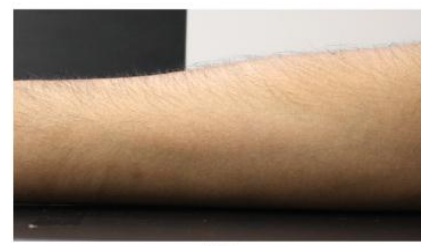

(b)

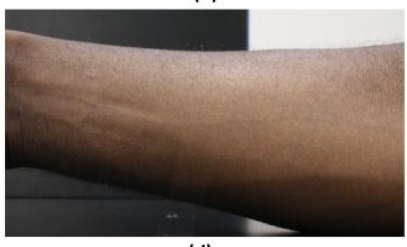

(d)
Figure 3. Four classes of skin tone. a) Fair, b) Light brown, c) Dark Brown and d) Dark.

As a comparison, Fig. 4 presents the same subjects viewed in NIR range from 740 to $980 \mathrm{~nm}$ (mean image). One can see that the veins are more visible. This figure also shows that even in NIR range, it is difficult to view the veins of subjects with darker skin tones. The next section will detail 


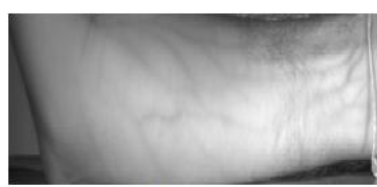

(a)

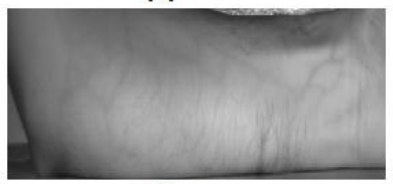

(c)

Figure 4. NIR image for each class. a) Fair, b) Light brown, c) Dark Brown and d) Dark.

the reflectance extraction of the skin and veins of the subjects from the multispectral images.

\section{THEORY AND CALCULATIONS}

Venous NIR Imaging works on the principles of light propagation, absorption, reflection and scattering in the different layers of skin. In the low absorption window, as illustrated by Fig. 1, light penetrates deeper inside the skin tissues allowing better visualization of veins.

As illustrated in Fig. 5, subcutaneous veins lie in the third layer of skin named hypodermis, on an average depth of 2 to $4 \mathrm{~mm}$, depending on the specific physical characteristics of a person and the location of skin on the body. For example, the skin on the back is much thicker as compared to the skin on the forearm.

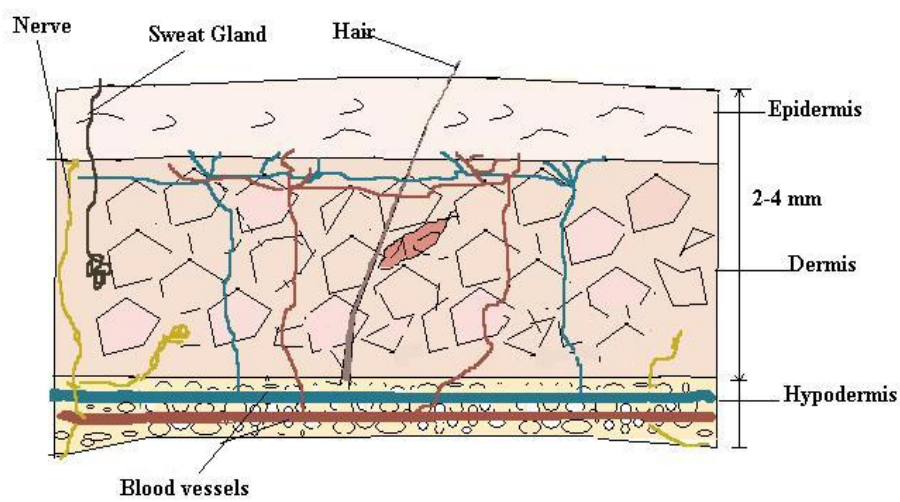

Figure 5. Simplified skin anatomy, depicting the three layers of human skin

On the basis of absorption and reflectance spectra of skin tissues and due to de-oxygenated hemoglobin (HbR), veins appear darker as compared to skin tissues.

Depending on the type and power of the illumination used, image intensity results might differ for a same subject. The extraction of reflectance is required in order to get the spectral responses independent from illumination. In nature, every element reflects a specific amount of energy out of the total energy it has been subjected to. The reflectance is defined as follows:

$$
R(x, y, \lambda)=\frac{E_{\text {reflected }}(x, y, \lambda)}{E_{\text {received }}(x, y, \lambda)}
$$

With $\boldsymbol{R}(\boldsymbol{x}, \boldsymbol{y}, \lambda)$, the reflectance value at the specific point (x, y) at specific wavelength $\lambda . \boldsymbol{E}_{\text {received }}(\boldsymbol{x}, \boldsymbol{y}, \boldsymbol{\lambda})$ and $\boldsymbol{E}_{\text {reflected }}(\boldsymbol{x}, \boldsymbol{y}, \lambda)$ are the received and reflected energy by the material, respectively.

The raw measurement $\boldsymbol{I}(\boldsymbol{x}, \boldsymbol{y}, \boldsymbol{\lambda})$ by the multispectral imaging system at the point coordinates $(\mathrm{x}, \mathrm{y})$ at specific wavelength $\lambda$ can be described by the following formula:

$I(x, y, \lambda)=L(x, y, \lambda) S(x, y, \lambda) \times R(x, y, \lambda)+O(x, y, \lambda)$

With $\boldsymbol{L}(\boldsymbol{x}, \boldsymbol{y}, \boldsymbol{\lambda})$ the illumination, $\boldsymbol{S}(\boldsymbol{x}, \boldsymbol{y}, \lambda)$ the spectral response of the camera system and $\boldsymbol{O}(\boldsymbol{x}, \boldsymbol{y}, \boldsymbol{\lambda})$ the offset which incorporates the camera system dark current and the stray light [14].

The calibration of the imaging system is done by using white and black Lambertian surfaces (shown in Fig. 2), with known reflectance values $\boldsymbol{R}_{\boldsymbol{w}}(\boldsymbol{x}, \boldsymbol{y}, \boldsymbol{\lambda})=0.98$ and $\boldsymbol{R}_{\boldsymbol{B}}(\boldsymbol{x}, \boldsymbol{y}, \boldsymbol{\lambda})=0.05$ respectively. Using Eq. 2 , the intensity values of images of white and black reference surfaces $I_{W}(x, y, \lambda)$ and $I_{B}(x, y, \lambda)$ can be computed.

Since $\boldsymbol{L}(\boldsymbol{x}, \boldsymbol{y}, \boldsymbol{\lambda}), \boldsymbol{O}(\boldsymbol{x}, \boldsymbol{y}, \boldsymbol{\lambda})$ and $\boldsymbol{S}(\boldsymbol{x}, \boldsymbol{y}, \boldsymbol{\lambda})$ can be considered as constants for a certain wavelength $\lambda$, and pixel coordinates $(\mathrm{x}, \mathrm{y})$, the product of illumination and the spectral response of the system i.e. $L(x, y, \lambda) \times S(x, y, \lambda)$ can be determined for each wavelength by the following formula:

$$
L(x, y, \lambda) \times S(x, y, \lambda)=\frac{I_{W}(x, y, \lambda)-I_{B}(x, y, \lambda)}{R_{W}(x, y, \lambda)-R_{B}(x, y, \lambda)}
$$

By substituting the value of $\boldsymbol{L}(\boldsymbol{x}, \boldsymbol{y}, \lambda) \times \boldsymbol{S}(\boldsymbol{x}, \boldsymbol{y}, \boldsymbol{\lambda})$ in (2) the offset at each wavelength can be computed as follows:

$O(x, y, \lambda)=\frac{I_{B}(x, y, \lambda) \times R_{W}(x, y, \lambda)-I_{W}(x, y, \lambda) \times R_{B}(x, y, \lambda)}{R_{W}(x, y, \lambda)-R_{B}(x, y, \lambda)}$

Finally, $\quad \boldsymbol{L}(\boldsymbol{x}, \boldsymbol{y}, \boldsymbol{\lambda}) \times \boldsymbol{S}(\boldsymbol{x}, \boldsymbol{y}, \boldsymbol{\lambda}) \quad$ and $\boldsymbol{O}(\boldsymbol{x}, \boldsymbol{y}, \boldsymbol{\lambda})$ are replaced in (2) in order to obtain reflectance value for each image taken from the setup. The formula is defined as:

$\boldsymbol{R}(\boldsymbol{x}, \boldsymbol{y}, \boldsymbol{\lambda})=$

$\frac{\left\{I(x, y, \lambda)-I_{B}(x, y, \lambda)\right\} \times R_{W}(x, y, \lambda)-\left\{I(x, y, \lambda)-I_{W}(x, y, \lambda)\right\} \times R_{B}(x, y, \lambda)}{I_{W}(x, y, \lambda)-I_{B}(x, y, \lambda)}$

With this equation spectral reflectance images can be extracted for each wavelength.

\section{RESULTS AND ANALYSIS}

In order to characterize the reflectance of veins and skin in function of the different skin tone subjects, rectangular Regions of Interest (ROIs) have been selected for each subject as illustrated in Fig. 6. The average values in the 


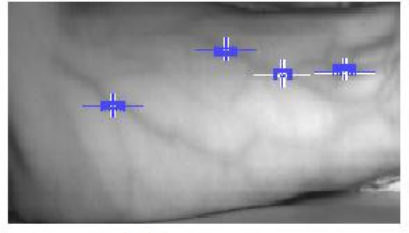

(a)

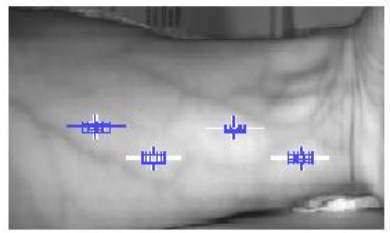

(b)
Figure 6. Selection of ROIs for a) veins and b) skin tissues for a light brown skin tone subject from the image in the NIR range (740-940nm)

selected ROIs are computed to obtain the skin tissues and veins reflectance at each wavelength.

The spectral reflectance of skin and veins for four classes of skin tone are shown in Fig. 7. The spectral response seems to be different for each different skin tone that underlines the necessity to use multiple illuminants (with different wavelengths) to robustly extract the veins.

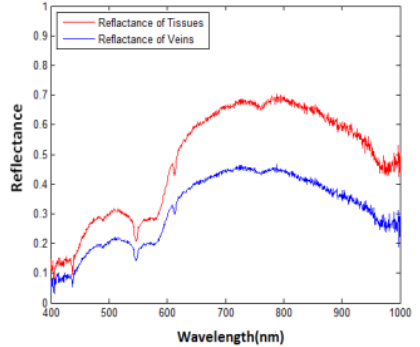

(a)

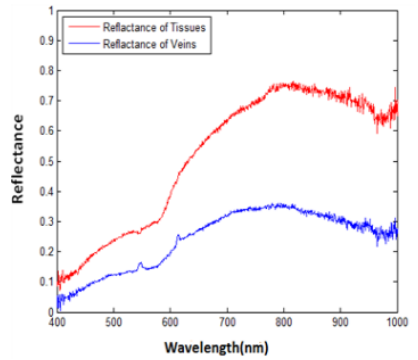

(c)

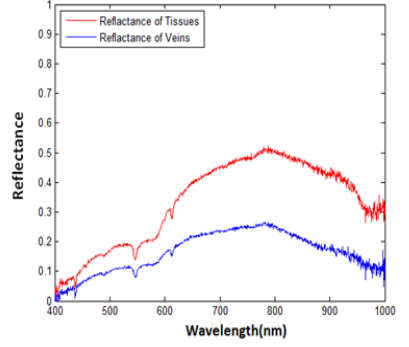

(b)

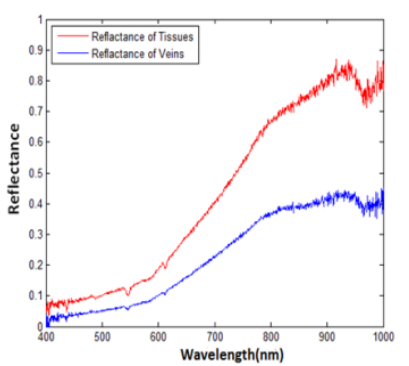

(d)
Figure 7. Reflectance plots of skin Tissues and Veins for four classes of skin a) Fair, b) Light Brown, c) Dark Brown and d) Dark.

To facilitate the extraction of veins, a high contrast between veins and skin is required. The choice of the multiple illuminants will then be conditioned by the maximum spectral contrast obtained for the different skin tones. Reflectance contrast values between veins and skin tissues for wavelength and class of skin tones have been computed. Fig. 8 exhibits the reflectance contrasts obtained for each skin tone. It seems that the range of high value of contrast differs from one skin tone to others. For the dark skin tone, the peak values lie in the range of 920 to $950 \mathrm{~nm}$. For dark brown tone, the range is around 830 to $900 \mathrm{~nm}$. For fair and light brown skin types, a wide range of wavelengths 790 to $870 \mathrm{~nm}$ is obtained. This might be due to the similarity of subjects having fair and light brown skin. In future data set will be increased, especially for the fair and light brown skin types, in order to obtain clearer classification of these skin types.

In order to select the LEDs as illuminant for the imaging system, we have selected the five optimum center wavelengths based on the commercial availability of the products and their characteristics (power, radiation angle and size). The blue vertical lines at $830,850,870,890$ and $950 \mathrm{~nm}$ in the Fig. 8 show the center wavelengths of selected LEDs. On the basis of this selection, we anticipate that we will get the maximum veins/tissue contrast using these LEDs for our veins localization system, no matter which skin type the subject has. In future work, our goal is to design an automated system, which can measure the skin tone on the basis of reflectance value for a particular subject. This system will be able to select the most suitable combination of wavelength for a particular skin type, in order to get the maximum vein/tissue contrast.

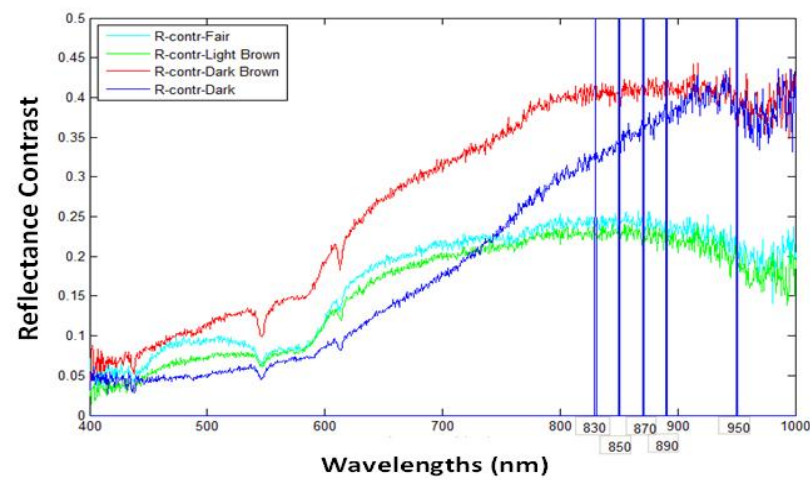

Figure 8. Reflectance contrast plot for four classes of skin tone, Fair, Light Brown, Brown and Dark.

\section{CONCLUSION}

In this paper we have investigated the spectral response of veins and skin tissues as a function of skin tone. From the multispectral images obtained for different subjects, reflectance graph of veins and skin tissues have been extracted for each skin tone. On the basis of the reflectance contrast, a pre-selection of illuminant LEDs to get best contrast for each class of skin tone has been proposed. Future work will investigate the automatic selection of illuminants (wavelengths) combination to optimally obtain high contrast images for each class of skin tone [15]. The database of subjects will be increased in order to better represent the different classes of skin tone. Algorithms to extract automatically the veins from images will be studied.

\section{REFERENCES}

[1] Barton A, Danek G, Johns P, Coons M., "Improving patient outcomes through CQI: Vascular access planning", Journal of Nursing Care Quality.;13(2):p 77-85. December, 1998. 
[2] Hadaway, Lynn Med "Infiltration and Extravasation"AJN, American Journal of Nursing: Volume 107 - Issue 8 - p 64-72August 2007.

[3] Katsogridakis YL, Seshadri R, Sullivan C, Waltzman ML. "Veinlite transillumination in the pediatric emergency department: a therapeutic interventional trial". Pediatric Emergency Care. Volume 24, Number 2, February 2008.

[4] Vincent Paquit, "Multispectral Imaging and 3D modeling for quantitative estimating subcutaneous blood vessels" $\mathrm{PhD}$ thesis, December 2008, University of Burgundy, France.

[5] T. Vo-Dinh, "Biomedical Photonics Handbook", CRC Press, Boca Raton, 2003.

[6] Sun Y, Jiang H, O'Neill BE, "Photoacoustic Imaging: An Emerging Optical Modality in Diagnostic and Theranostic Medicine”. J Biosens Bioelectron 2:108; September 2011.

[7] DiMarzio, C. A. and Murray, T.W.“ Medical imaging techniques combining light and ultrasound". Subsurface Sensing Technologies and Applications, 4(4):289-309; October 2003.

[8] Niederhauser,J. "Real-Time Biomedical Photoacoustic Imaging", $\mathrm{PhD}$ thesis 2004, Swiss Federal Institute of Technology Zurich, Switzerland.

[9] Gary Strangman, David A. Boas, and Jeffrey P. Sutton., "NonInvasive Neuroimaging Using Near-Infrared Light" Biol Psychiatry, 2002; 52:679-693, October 2002.

[10] Zeman, H., Lovhoiden, G., Vrancken, C., and Danish, R. "Prototype vein contrast enhancer." Optical Engineering, 44, 086401 August 2005.

[11] Zeman, H. D. "Projection of subsurface structure onto an object's surfaces". U.S. Patent N0. 20060122515. 2006.

[12] Paquit, V. C., Price, J. R., Mériaudeau, F., Tobin, K. W., and Ferrell, T. L., "Combining Near-Infrared Illuminants to Optimize Venous Imaging," Proceedings of the SPIE Medical Imaging 2007: Visualization, Image-Guided Procedures, and Display, San Diego, California, USA, February 2007.

[13] I. Nishidate, T. Maeda, Y. Aizu, and K. Niizeki,"Visualizing depth and thickness of a local blood region in skin tissue using diffuse reflectance images.," J Biomed Opt, vol. 12, no. 5, pp. 054006, September 2007.

[14] Z. Pan, G. Healey, M. Prasad, and B. Tromberg, "Face recognition in hyperspectral images," IEEE Transactions on

Pattern Analysis and Machine Intelligence 25(12), pp. 15521560,December 2003.

[15] Vincent Paquit, Jeffery Price, Kenneth Tobin and Fabrice Meriaudeau, "3D and multispectral imaging for subcutaneous veins detection", Optics Express, 17 (14), pp. 11360-11365, July 2009 . 